\title{
ESTUDO COMPARATIVO CONTROLADO COM EMPREGO DE BENZNIDAZOLE, NIFURTIMOX E PLACEBO, NA FORMA CRÔNICA DA DOENÇA DE CHAGAS, EM UMA ÁREA DE CAMPO COMTRANSMISSÃO INTERROMPIDA. I. AVALIAÇÃO PRELIMINAR
}

\author{
José Rodrigues Coura, Laércio Luiz de Abreu†, Henry Percy \\ Faraco Willcox e Walter Petana
}

\begin{abstract}
Foi realizado um estudo controlado para avaliar a eficácia terapêutica e a tolerância do nifurtimox e do benznidazole em pacientes com a doença de Chagas crônica. Todos os pacientes tinham as reações de imunofluorescência e fixação do complemento positivas para anticorpos anti-T. cruzi e pelo menos dois xenodiagnósticos positivos em três realizados, antes do tratamento, e foram submetidos a exames clínicos, eletrocardiográficos e radiográficos do coração e do esôfago. De 77 pacientes estudados, 27 foram tratados com nifurtimox, 26 com benznidazole, ambos na dose de $5 \mathrm{mg} / \mathrm{kg} /$ dia, durante 30 dias consecutivos, e 24 receberam um placebo em comprimidos semelhantes aos do benznidazole. Dos 77 pacientes, 64 (83,1\%) completaram o tratamento: 23 (88,4\%) com benznidazole, 19 (70,3\%) com nifurtimox e 22 (91,6\%) com placebo. Os pacientes foram avaliados clinicamente, sorologicamente $e$ parasitologicamente (seis xenodiagnósticos no período de um ano após o tratamento). o grupo do benznidazole mostrou apenas 1,8\% de xenodiagnósticos positivos póstratamento, o grupo do nifurtimox 9,6\% e o do placebo 34,3\%. Todas as reações sorológicas continuaram positivas e não bouve alterações clínicas, eletrocardiográficas ou radiológicas um ano após o tratamento.
\end{abstract}

Palavras-chaves: Doença de Chagas. Tratamento específico. Benznidazole. Nifurtimox.

São poucos os estudos controlados sobre a terapêutica específica da doença de Chagas crônica, particularmente em áreas de campo, onde o acompanhamento dos pacientes tornase mais difícil, por falta de condições laboratoriais e de profissionais capacitados para fazê-lo adequadamente.

Alguns estudos sobre tolerância e eficácia do nifurtimox e do benznidazole em pacientes nas fases aguda e crônica da doença de Chagas foram realizados no Brasil e em outros países do nosso continente 6121315161720212829303132 , mostrando importantes efeitos colaterais com as duas drogas e indícios de cura parasitológica em torno de $60 \%$ nos casos agudos, porém pouco expressivos nos casos crônicos.

\footnotetext{
Departamentos de Medicina Preventiva da Universidade Federal do Rio de Janeiro e de Medicina Tropical do Instituto Oswaldo Cruz (FIOCRUZ), Rio de Janeiro, RJ. t In memorian.

Endereço para correspondência: Prof. José Rodrigues Coura. Dept ${ }^{\underline{o}}$ de Medicina Tropical/FIOCRUZ. Av. Brasil 4365, 21045 900, Rio de Janeiro, RJ

Recebido para publicação em 06/03/96.
}

O controle de cura, no entanto, naqueles estudos variou muito de um para outro, o que dificulta a sua comparação. A maioria deles baseia-se na avaliação seriada de duas ou mais reações sorológicas e em repetidos xenodiagnósticos. Cançado e cols ${ }^{14}$ estabeleceram as "bases para a avaliação do tratamento específico da doença de Chagas humana segundo a parasitemia", indicando para tratamento pacientes com provas sorológicas positivas e com pelo menos $60 \%$ de xenodiagnósticos positivos em no mínimo seis realizados com 48 ninfas de $T$. infestans ou $P$. megistus, internação dos pacientes durante o tratamento, e seguimento ambulatorial com realização de xenodiagnósticos e reações sorológicas de 15 em 15 dias, durante pelo menos um ano, o que naturalmente é impossível de ser realizado em campo.

Mais recentemente, novas perspectivas foram abertas para o controle de cura parasitológica da doença de Chagas, através da dosagem de anticorpos líticos ${ }^{22}$, do 
Coura JR, Abreu LL, Willcox HPF, Petana W. Estudo comparativo controlado com emprego de benznidazole, nifurtimox e placebo, na forma crônica da doença de Chagas, em uma área de campo com transmissão interrompida. I. Avaliaçẫo preliminar. Revista da Sociedade Brasileira de Medicina Tropical 30:139-144, mar-abr, 1997.

desenvolvimento de técnica de hemocultura mais sensível ${ }^{25}$ e da reação de polimerase em cadeia (PCR $)^{11}$. Por outro, lado apesar de não ter surgido nenhuma nova droga de atividade expressiva para o tratamento específico da infecção chagásica nos últimos 30 anos, quando os primeiros testes clínicos foram iniciados com o nifurtimox em 1965 e com o benznidazole em 1971, uma nova consciência vem se formando entre os pesquisadores da área sobre a necesssidade de reabilitação do tratamento específico da doença, como tem sido demonstrado pela análise desse tema em reuniões sobre Pesquisa Aplicada em Doença de Chagas, realizadas em Araxá (1986) e Uberaba (1994) e recentemente (fevereiro de 1996) no Ministério da Saúde.

A impressão de que o nifurtimox e o benznidazole não tinham ação curativa nem efeito sobre a evolução da doença de Chagas crônica, a qual seria predominantemente imuno-dependente, aliada a dificuldades relacionadas ao tratamento prolongado com drogas tóxicas e potencialmente carcinogênicas ${ }^{33}{ }^{34}$ e a restrições na avaliação do controle de cura (devido a baixa parasitemia e manutenção da positividade das reações sorológicas após o tratamento), desestimularam o tratamento da doença nessa fase, e até mesmo a publicação de resultados de tratamentos realizados.

Com a "reabilitação" do parasita como principal responsável pela patogênese da doença, mesmo na fase crônica ${ }^{23}$, e com a melhoria nas técnicas de diagnóstico parasitológico e de controle de cura e a comprovação da melhor evolução de casos crônicos tratados com benznidazole ${ }^{36} \mathrm{em}$ relação a casos não tratados, volta o interesse pelo uso dessas drogas, pela reavaliação e publicação de resultados de casos anteriormente tratados e, talvez, pelo emprego de novos esquemas com doses mais baixas e prolongadas, em tratamentos sucessivos.

O presente trabalho representa exatamente um estudo de campo com emprego do benznidazole e do nifurtimox, este em baixas doses, em dois grupos de pacientes chagásicos crônicos, controlados por um terceiro grupo que recebeu placebo. Este trabalho refere-se exclusivamente ao benznidazole e ao nifurtimox e não pretende revisar outras drogas de uso mais recente ou drogas utilizadas no passado ${ }^{818} 192027$, nem avaliar perspectivas futuras do tratamento específico?.

\section{MATERIAL E MÉTODOS}

Entre 158 pacientes chagásicos crônicos residentes nos municípios de Pains e Iguatama, no Oeste de Minas Gerais, foram selecionados 80 para tratamento com benznidazole, nifurtimox ou placebo. Dos 80 pacientes selecionados, 77 concordaram em submeter-se ao tratamento e aos exames de controle, depois de informados sobre seus efeitos colaterais.

Seleção dos pacientes. Os pacientes selecionados para o tratamento tinham a reação de imunofluorescência em sangue colhido em papel de filtro (IFP) positiva para infecção chagásica, confirmada por essa reação e pela fixação de complemento no soro (IFS, RFC) e por pelo menos dois xenodiagnósticos positivos para T. cruzi em três realizados, em meses consecutivos antes do tratamento. Dos 80 pacientes, $51(63,7 \%)$ tiveram os três xenodiagnósticos positivos e 29 (36,2\%) tiveram dois xenodiagnósticos positivos nos três realizados, portanto, foram considerados com alta parasitemia. Todos os pacientes eram examinados clinicamente, submetidos ao eletrocardiograma com as 12 derivações clássicas e à radiografia de tórax de frente e perfil com esôfago contrastado.

Os xenodiagnósticos foram realizados aplicando-se 40 ninfas de T. infestans de 3우은 estádios, em jejum de 15 a 20 dias, distribuídas igualmente em quatro caixas de madeira, duas em cada antebraço, durante 30 minutos. A primeira leitura dos xenodiagnósticos foi realizada no $30^{\circ}$ dia após o repasto, examinando-se individualmente as fezes de cada triatomíneo, obtidas por leve compressão abdominal do inseto, colocadas em duas lâminas em gota de PBS pH 7,2, homogeinizadas, cobertas com lamínulas de 22 × $22 \mathrm{~mm}$ e examinadas em toda a sua extensão em microscópio com aumento de 400 vezes. Quando negativos, os triatomíneos eram realimentados com sangue de galinha e reexaminados 30 dias depois pela mesma técnica. Se ainda negativos, retirava-se o conteúdo intestinal por dissecção e reexaminava-se, dando-se o exame como concluído.

Constituição dos grupos e tratamento dos pacientes. Foram constituídos três grupos de pacientes com média de idade, sexo e forma clínica semelhantes entre si. A droga foi administrada para cada grupo de forma aleatória e o médico que a ministrava sabia 
Coura JR, Abreu LL, Willcox HPF, Petana W. Estudo comparativo controlado com emprego de benznidazole, nifurtimox e placebo, na forma crônica da doença de Chagas, em uma área de campo com transmissão interrompida. I. Avaliação preliminar. Revista da Sociedade Brasileira de Medicina Tropical 30:139-144, mar-abr, 1997.

apenas que o grupo I devia tomar a droga $\mathrm{A}$, o II a droga B e o III a droga C.

Grupo I: constituído de 26 pacientes, foi sorteado para tomar a droga A (benznidazole) na dose de $5 \mathrm{~m} / \mathrm{kg} /$ dia em duas tomadas, de 12 em 12 horas, durante 30 dias.

Grupo II: constituído de 27 pacientes, foi sorteado para tomar a droga B (nifurtimox), também na dose de $5 \mathrm{~m} / \mathrm{kg} /$ dia em duas tomadas, de 12 em 12 horas, durante 30 dias. A dose mais baixa foi propositadamente determinada devido a nossa experiência anterior de intolerância digestiva e abandono de tratamento por pacientes de ambulatório hospitalar tratados com essa droga.

Grupo III: constituído por 24 pacientes, foi sorteado para tomar a droga C (placebo de amido em comprimidos semelhantes aos do benznidazole), em duas tomadas, de 12 em 12 horas, durante 30 dias.

Os pacientes recebiam o medicamento para tratamento semanal e semanalmente eram revisto pelo médico, que os examinavam e anotava as queixas e efeitos colaterais, durante todo o período do tratamento e até 15 dias após.

Controle de cura. O controle de cura foi feito no $1^{\circ}, 2^{\circ}$ e $3^{\circ}$ meses após o tratamento, quando os pacientes eram reexaminados $\mathrm{e}$ submetidos ao xenodiagnóstico com a mesma técnica anteriormente descrita. No $12^{\mathrm{o}}$ mês após o tratamento os pacientes eram reexaminados e submetidos a três xenodiagnósticos com intervalos de 15 dias, totalizando seis xenodiagnósticos, após o tratamento. Também nessa última revisão foi colhido sangue por punção venosa e realizadas as reações de imunofluorescência e fixação do complemento para pesquisa de anticorpos anti-T. cruzi.

Testes estatísticos. Para avaliação do abandono de tratamento devido a efeitos colaterais foi utilizado o teste de Fischer (para pequenos números) e para avaliação do controle de cura pelo xenodiagnóstico, foi utilizado o teste do qui-quadrado (Tabela 1 ).

\begin{tabular}{|c|c|c|c|c|c|c|}
\hline \multirow[t]{2}{*}{ Grupos de drogas } & \multirow{2}{*}{$\begin{array}{l}\text { Pacientes } \\
\text { tratados }\end{array}$} & \multirow{2}{*}{$\begin{array}{c}\text { Abandono de } \\
\text { tratamento }\end{array}$} & \multirow[b]{2}{*}{$\%^{*}$} & \multicolumn{3}{|c|}{ Xenodiagnósticos ** } \\
\hline & & & & aplicados & positivos & $\%$ \\
\hline I (benznidazole) & 26 & 3 & 11,5 & 110 & 2 & 1,8 \\
\hline II (nifurtimox) & 27 & 8 & 29,6 & 83 & 8 & 9,6 \\
\hline III (placebo & 24 & 2 & 8,3 & 67 & 23 & 34,3 \\
\hline Total & 77 & 13 & 16,8 & 260 & 43 & 16,5 \\
\hline
\end{tabular}

${ }^{*}$ Teste de Fischer; ${ }^{* *}$ Qui-quadrado.

\section{RESULTADOS}

Dos 77 pacientes, $64(83,1 \%)$ completaram o tratamento, $23(88,4 \%)$ do grupo I, 19 $(70,3 \%)$ do grupo II e $22(91,6 \%)$ do grupo III. Oito pacientes do grupo II, ou seja, 29,6\% abandonaram o tratamento antes de completálo, contra $3(11,5 \%)$ do grupo I e $2(8,3 \%)$ do grupo III. Os efeitos colaterais, apesar da baixa dose, foram mais intensos e diversificados nos pacientes tratados com nifurtimox, principalmente a intolerância digestiva, as parestesias e as manifestações neuropsíquicas, enquanto que com o benznidazole as manifestações mais freqüentes foram a erupção cutânea, a neuropatia periférica e os distúrbios gastrointestinais, porém, discretos ou moderados. Também no grupo placebo sete pacientes $(29,1 \%)$ apresentaram manifestações do tipo aerofagia, plenitude pós-prandial, anorexia, cefaléia, sonolência e lassidão, manifestações possivelmente de ordem psíquica por saberem que estavam recebendo um medicamento.

Dos 110 xenodiagnósticos realizados no grupo I após o tratamento, apenas dois $(1,8 \%)$ foram positivos, um deles no $2^{\mathrm{o}}$ e o outro no $12^{\mathrm{o}}$ mês, enquanto dos 83 xenodiagnósticos realizados no grupo II, oito $(9,6 \%)$ foram positivos, sete dos quais nos três primeiros meses após o tratamento e um no $12^{\circ}$ mês. No grupo placebo de 67 xenodiagnósticos póstratamento realizados, $23(34,3 \%)$ foram positivos. As reações sorológicas continuavam positivas em todos os pacientes de todos os grupos no $12^{\circ}$ mês após o tratamento. 
Coura JR, Abreu LL, Willcox HPF, Petana W. Estudo comparativo controlado com emprego de benznidazole, nifurtimox e placebo, na forma crônica da doença de Chagas, em uma área de campo com transmissão interrompida. I. Avaliaçẫo preliminar. Revista da Sociedade Brasileira de Medicina Tropical 30:139-144, mar-abr, 1997.

Os exames clínicos, radiológicos e eletrocardiográficos dos pacientes não sofreram alterações após o tratamento.

O abandono de tratamento devido a efeitos colaterais foi significativamente maior no grupo tratado com o nifurtimox do que o tratado com o benznidazole em relação ao placebo $(\mathrm{p}<0,05)$.

Em relação ao controle de cura avaliado pelo xenodiagnóstico, a eficácia do benznidazole foi 18 vezes maior que o placebo e 5 vezes maior que o nifurtimox, sendo este apenas 3,5 vezes maior que o placebo (Tabela 1 ). Devese considerar, entretanto, as baixas doses de nifurtimox utilizadas.

\section{DISCUSSÃO}

Com relação aos efeitos colaterais, ficou claro neste trabalho, que o nifurtimox, mesmo em doses baixas de $5 \mathrm{mg} / \mathrm{kg} / \mathrm{dia}$, quando a dose recomendada é de 8 a $10 \mathrm{mg}$, são bem mais intensos e diversificados do que com o benznidazole, uma vez que $29,6 \%$ dos pacientes tratados com aquela droga abandonaram o tratamento por intolerância, enquanto com o benznidazole apenas $3(11,5 \%)$ dos pacientes desistiram do tratamento. É interessante notar que $29,1 \%$ dos casos que receberam o placebo, apresentaram manifestações gerais de baixa intensidade e importância, no entanto, mesmo assim, dois deles $(8,3 \%)$ abandonaram o tratamento.

Quanto à eficácia, embora não possam rigorosamente serem comparadas as duas drogas, porque o nifurtimox foi usado em doses mais baixas do que seria recomendada, o benznidazole foi bem mais eficaz, uma vez que apenas dois xenodiagnósticos póstratamento $(1,8 \%)$ foram positivos, contra 8 $(9,6 \%)$ nos pacientes tratados com nifurtimox e $34,3 \%$ com o placebo, que equivale, de fato, à positividade do xenodiagnóstico em pacientes não tratados. O mais importante no caso é que sete dos oito pacientes tratados com nifurtimox que tiveram os xenodiagnósticos positivos, a positividade ocorreu nos três primeiros meses após o tratamento, portanto, mostrando ineficácia absoluta, enquanto que com o benznidazole o efeito supressivo da parasitemia ainda persistia um ano após o tratamento na maioria dos casos. Essa falha do nifurtimox pode estar relacionada à baixa dose utilizada ou à resistência da cepa do T. cruzi, entretanto, sabe-se que a resistência quase sempre é cruzada ${ }^{12410}$.

A persistência da positividade das reações sorológicas após o tratamento pode ter duas interpretações: a) os pacientes não foram curados parasitologicamente; b) a presença de células de "memória" e ou de restos parasitários estariam mantendo a anticorpogênese ${ }^{3}$. Os métodos mais recentes de diagnóstico como a dosagem dos anticorpos líticos, o PCR e a detecção de antígenos parasitários, poderão vir a solucionar esta incógnita.

O problema mais importante no tratamento da fase crônica da doença de Chagas é sabermos se o paciente se beneficia ou não na evolução de sua doença, particularmente os casos da forma indeterminada. Alguns autores $^{26}{ }^{35}$ acham que não, no entanto, trabalho recente de Viotti e cols ${ }^{36}$, indica claramente esse benefício. Recomenda-se, portanto, a revisão retrospectiva de casuísticas tratadas anteriormente, como a deste trabalho, e o desenvolvimento de novos projetos, para estudos prospectivos controlados, inclusive com tratamentos repetidos, como sugerido nesta discussão, com essas e com novas drogas.

\section{SUMMARY}

A controled clinical trial was carried out to evaluate the therapeutic efficacy and tolerance of nifurtimox and benznidazole in patients with chronic Chagas' disease. All patients had immunofluorescence and complement fixation reactions positives for $\mathrm{T}$. cruzi antibodies and at least two xenodiagnoses positives in three performed before treatment, and they were submitted to clinical examinations, ECG and X-ray of the heart and esophagus. Of 77 patients studied, 27 were treated with nifurtimox and 26 with benznidazole in the dosage of $5 \mathrm{~m} / \mathrm{kg} /$ day for 30 consecutives days, and 24 received a placebo in tablets similar to benznidazole. From the 77 patients, 64 (83,1\%) accomplished the treatment: $23(88,4 \%)$ with benznidazole, 19 (70,3\%) with nifurtimox and 22 (91,6\%) with placebo. The patients were evaluated, clinically, serologically and parasitologically (six xenodiagnoses within one year after treatment). The benznidazole group showed only 1,8\% of positive xenodiagnoses post-treatment, the nifurtimox 9,6\% and the placebo 34,3\%. All serologic reations continued positives and there were no clinical, ECG or X-ray changes one year after treatment.

Key-words: Chagas' disease. Specific treatment. Benznidazole. Nifurtimox. 
Coura JR, Abreu LL, Willcox HPF, Petana W. Estudo comparativo controlado com emprego de benznidazole, nifurtimox e placebo, na forma crônica da doença de Chagas, em uma área de campo com transmissão interrompida. I. Avaliaçẫo preliminar. Revista da Sociedade Brasileira de Medicina Tropical 30:139-144, mar-abr, 1997.

\section{REFERÊNCIAS BIBLIOGRÁFICAS}

1. Andrade SG, Figueira RM. Estudo experimental sobre a ação terapêutica da droga R0-7-1051 na infecção por diferentes cepas do Trypanosoma cruzi. Revista do Instituto de Medicina Tropical de São Paulo 19:335-341, 1977.

2. Andrade SG, Figueira RM, Carvalho ML, Gorini DF. Influência da cepa do Trypanosoma cruzi na resposta à terapêutica experimental pelo Bay 2502 (Resultados de tratamento a longo prazo). Revista do Instituto de Medicina Tropical de São Paulo 17:380-389, 1975.

3. Andrade SG, Freitas LAR, Peyrol S, Pimentel AR, Sadigursky M. Experimental chemotherapy of Trypanosoma cruzi infection: persistence of parasite antigens and positive serology in parasitologically cured mice. Bulletin of the World Health Organization 69:191-197, 1991.

4. Andrade SG, Rassi A, Magalhães JB, Ferrioli-Filho F, Luquetti AO. Specific chemotherapy of Chagas disease: a comparison between the response in patients and experimental animals inoculated with the same strains. Transactions of the Royal Society of Tropical Medicine and Hygiene 86:624-626, 1992.

5. Bellotti G, Pileggi F. Trypanosoma cruzi e cardiopatia chagásica crônica. Arquivos Brasileiros de Cardiologia 61:203-205, 1993.

6. Boainain E. Tratamento etiológico da doença de Chagas na fase crônica. Tese. Faculdade de Medicina da Universidade Federal de Goiás, Goiânia, GO, 1977.

7. Bocca-Tourres CL. La enfermedad de Chagas en período agudo y su tratamiento con el Bay 2502. Boletin Chileno de Parasitologia 24:24-27, 1969.

8. Brener Z. Contribuição ao estudo da terapêutica experimental da doença de Chagas. Tese. Faculdade de Odontologia e Farmácia da Universidade Federal de Minas Gerais, Belo Horizonte, MG, 1961.

9. Brener Z. Recent advances in the chemotherapy of Chagas' disease. Memórias do Instituto Oswaldo Cruz 79 (supl):149-155, 1984.

10. Brener Z, Costa CAG, Chiari C. Diferences in the susceptibility of Trypanosoma cruzi strains to active chemotherapeutic agents. Revista do Instituto de Medicina Tropical de São Paulo 18:450-455, 1976.

11. Britto C, Cardoso MA, Vannic MM, Moreno AH, Xavier SS, Oelemann W, Santoro A, Pirmez C,
Morel CM, Winker P. Polimerase Chain Reaction. Detection of Trypanosoma cruzi in human blood samples as a tool for diagnosis and treatment evaluation. Parasitology 110:241-247, 1995.

12. Cançado JR. Tratamento específico. In: Cançado JR, Schuster M (eds) Cardiopatia chagásica. Imprensa Oficial do Estado de Minas Gerais, Belo Horizonte, Brasil, p. 327-355, 1985.

13. Cançado JR, Marra UD, Lopes M, Mourão O, Faria CAF, Álvares JM, Salgado AA. Toxicidad y valor terapeutico del Bay 2502 en la enfermedad de Chagas crónica en tres esquemas posológicos. Boletin Chileno de Parasitologia 24:28-32, 1969.

14. Cançado JR, Marra UD, Mourão OG, Álvares JM, Oliveira JPM, Machado JR, Salgado AA. Bases para a avaliação do tratamento específico da doença de Chagas humana segundo a parasitemia. Revista da Sociedade Brasileira de Medicina Tropical 7:155-166, 1973.

15. Cançado JR, Salgado AA, Batista SM, Chiari C. Segundo ensaio terapêutico com o nifurtimox na doença de Chagas. Revista Goiana de Medicina 22:203-233, 1976.

16. Cançado JR, Salgado AA, Marra UD, Álvares JM. Ensaio terapêutico clínico na doença de Chagas crônica com o nifurtimox em três esquemas de duração prolongada. Revista do Instituto de Medicina Tropical de São Paulo 17:111-125, 1975.

17. Cerisola JA, Lugones H, Rabinovich LB.Tratamiento de la Enfermedad de Chagas. Talleres Gráficos Elías Porter SRL. Buenos Aires, Argentina, 75p, 1972.

18. Coura JR, Ferreira LF, Silva JR. Experiências com a nitrofurazona na fase crônica da doença de Chagas. O Hospital 62:957-964, 1962.

19. Coura JR, Silva JR. Aspectos atuais do tratamento da doença de Chagas. Arquivos Brasileiros de Medicina 51:283-290, 1961.

20. Ferreira HO. Ensaio terapêutico-clínico com o benzonidazol na doença de Chagas. Revista do Instituto de Medicina Tropical de São Paulo 18:357-364, 1976.

21. Ferreira HO. Tratamento da forma indeterminada da doença de Chagas com nifurtimox e benzonidazol. Revista da Sociedade Brasileira de Medicina Tropical 23:209-211, 1990.

22. Galvão LMC, Nunes RMB, Cançado JR, Brener Z, Krettli AU. Lityc antibody titre as a mean of assessing cure after treatment of Chagas' disease: 
Coura JR, Abreu LL, Willcox HPF, Petana W. Estudo comparativo controlado com emprego de benznidazole, nifurtimox e placebo, na forma crônica da doença de Chagas, em uma área de campo com transmissão interrompida. I. Avaliação preliminar. Revista da Sociedade Brasileira de Medicina Tropical 30:139-144, mar-abr, 1997.

a 10 years follow-up study. Transactions of the Royal Society of Tropical Medicine and Hygiene 87:220-223, 1993.

23. Higuchi ML, Brito T, Reis MM, Brbosa A, Bellotti G, Pereira-Barreto AC, Pileggi F. Correlation between Trypanosoma cruzi parasitism and myocardial inflamatory infiltrate in human chronic chagasic myocarditis: ligth microscopy and immunohistochemical findings. Cardiovascular Pathology 2:101-106, 1993

24. Krettli AU, Cançado JR, Brener Z. Criterion of cure of human Chagas' disease after specific chemotherapy: recent advances. Memórias do Instituto Oswaldo Cruz 79 (supl):157-164, 1984.

25. Luz ZMP, Coutinho MG, Cançado JR, Krettli AU. Hemocultura: técnica sensível na detecção do Trypanosoma cruzi em pacientes chagásicos na fase crônica da doença de Chagas. Revista da Sociedade Brasileira de Medicina Tropical 27:143$148,1994$.

26. Macedo V, Silveira CA. Perspectivas da Terapêutica Específica na doença de Chagas. Experiências na forma indeterminada. Revista da Sociedade Brasileira de Medicina Tropical 20 (supl II):M-24M-26, 1987.

27. Prata A. Estado atual da terapêutica específica da doença de Chagas. Revisão dos medicamentos até hoje utilizados. Revista Goiana de Medicina 9 (supl):109-124, 1963.

28. Prata A, Macedo V, Porto G, Santos I, Cerisola JA, Silva N. Tratamento da doença de Chagas pelo nifurtimox (Bayer 2502). Revista da Sociedade Brasileira de Medicina Tropical 9:297-307, 1975.

29. Rassi A, Ferreira, HO. Tentativa de tratamento específico da fase aguda da doença de Chagas com nifurtimox em esquemas de duração prolongada. Revista da Sociedade Brasileira de Medicina Tropical 5:235-262, 1971.
30. Rassi A, Luquetti AO. Therapy of Chagas'disease. In: Wendel S, Brener Z, Camargo ME, Rassi A (eds) Chagas' disease (American Trypanosomiasis): its impact on transfusion and clinical medicine. Cartgraf Editora Ltda, ISBT Brazil, 92, p.237-247, 1992.

31. Rubio M, Donoso F. Enfermedad de Chagas en niños y tratamiento con Bay 2502. Boletin Chileno de Parasitologia 24:43-48, 1969.

32. Schenone H, Concha L, Aranda R, Rojas A, Alfaro E. Experiencia terapêutica con el Bay 2502 en la infección chagásica crónica del adulto. Importância del uso adequado del xenodiagnóstico. Boletin Chileno de Parasitologia 24:66-69, 1969.

33. Teixeira ARL, Calixto MA, Rizzo LV, Córdoba JC, Teixeira ML. Cancerigênese em camundongos tratados com compostos nitroderivados. In: Resumos do XXI Congresso da Sociedade Brasileira de Medicina Tropical, São Paulo, SP, p.48-49, 1985

34. Teixeira ARL, Córdoba JC, Souto-Maior I, Solórzano E. Chagas' disease: lymphoma growth in in rabbits treated with benznidazole. The American Journal of Tropical Medicine and Hygiene 43:146-158, 1990.

35. Teixeira A, Tinoco D, Maneta L, Sales L, Barcelos G, Maurmo M, Pires L. Os perfis parasitológico, imunológico e eletrocardiográfico de chagásicos crônicos tratados com nitroarenos são indistinguíveis daqueles observados nos chagásicos não tratados. Revista da Sociedade Brasileira de Medicina Tropical 22 (supl II):110, 1989.

36. Viotti R, Vigliano C, Arment H, Segura E. Treatment of Chagas' disease with benznidazole: clinical and serologic evolucion of patients with long-term follow-up. American Heart Journal 127:151-162, 1994. 